\title{
ON THE ITERATION OF POLYNOMIALS OF DEGREE 4 WITH REAL COEFFICIENTS
}

\author{
P. BHATTACHARYYA and Y. E. ARUMARAJ
}

\section{Introduction}

The theory of iteration of a rational or entire function $f(z)$ of a complex variable $z$ deals with the sequence of natural iterates $f_{n}(z)$ defined by

$$
f_{0}(z)=z, \quad f_{n+1}(z)=f\left(f_{n}(z)\right), \quad n=0,1,2, \ldots .
$$

In the theory developed by Fatou [5,6] and Julia [8], the fundamental object of study is the set $F(f)$ of those points of the complex plane in no neighbourhood of which $\left\{f_{n}(z)\right\}$ forms a normal family in the sense of Montel. Unless $f(z)$ is a rational function of order 0 or 1 , the set $F(f)$ has the following properties, proved for rational functions in [5] and for entire functions in [6].

I. $F(f)$ is a nonempty perfect set.

II. $F\left(f_{n}\right)=F(f)$ for any integer $n \geqq 1$.

III. $F(f)$ is completely invariant under the mapping $z \rightarrow f(z)$, i.e. if $\alpha$ belongs to $F(f)$, then so do $f(\alpha)$ and every solution $\beta$ of $f(\beta)=\alpha$.

A fixpont $\alpha$ of order $n$ of $f(z)$ is a solution of $f_{n}(z)=z$; it has exact order $n$ if $f_{k}(\alpha)=\alpha$ for $k=n$, but not for any $k<n$, and in this case $f_{n}^{\prime}(\alpha)$ is called the multiplier of $\alpha$, provided $\alpha \neq \infty$. The fixpoint is called attractive, indifferent or repulsive according as the modulus of its multiplier is less than, equal to or greater than 1 , respectively. With this nomenclature we have:

An indifferent fixpoint $\alpha$ is called rationally indifferent if $f^{\prime}(\alpha)=e^{2 \pi i p}$, where $p$ is a rational number.

With this nomenclature we have:

IV a) Attractive fixpoints belong to the complement of $F(f)$.

b) Rationally indifferent fixpoints belong to $F(f)$.

c) The set $F(f)$ is identical with the closure of the set of repulsive fixpoints.

In their original work Fatou [5, 6] and Julia [8] have derived a number of general properties of the set $F(f)$. The structure of $F(f)$ depends on the function $f$. If $f$ is a polynomial, $F(f)$ depends on the coefficients of $f$ in a very complicated manner. Myrberg [9-13], Brolin [4], and Bhattacharyya and Arumaraj [3] have con- 
sidered the cases where $f$ is a polynomial of degree 2 and 3 with real coefficients.

In this paper we investigate the structure of $F(f)$ when $f$ is a polynomial of degree 4 with real coefficients.

Baker [1] has shown that for suitably chosen $k, F\left(k z e^{z}\right)$ is the whole plane. Bhattacharyya [2] has shown that $F\left(e^{z}-1\right)$ has inaccessible boundary points. Other examples are studied by Van Haselen [7] and Topffer [14].

Two polynomials $f(z)$ and $g(z)$ are said to be similar if there exist constants $a$ and $b$ such that $f(a z+b)=a g(z)+b$. Clearly, if $\alpha$ is a fixpoint of order $n$ of multiplier $k$ of $g(z)$, then $a \alpha+b$ is a fixpoint of $f(z)$ of the same order $n$ and same multiplier $k$.

The most general polynomial of degree 4 with real coefficients can easily be shown to be similar to

$$
f(z)=z^{4}+p z^{2}+r z+s, \quad p, r, s \text { reals. }
$$

By IV above it is enough if we consider this simpler polynomial to study the structure of $F(f)$ where $f$ is a polynomial of degree 4 with real coefficients.

We consider the following separate cases:

Case A. $f(z)=z^{4}+p z, \quad p$ real,

Case B. $f(z)=z^{4}+r, \quad r$ real,

Case C. $f(z)=z^{4}+p z^{2}, \quad p$ real,

Case D. $f(z)=z^{4} a-p z^{2}+\sqrt{p}, \quad p$ real,

Case E. $f(z)=z^{4}+p z^{2}+r z+s, p, q, r, s$ reals.

In Section 2 we give a detailed analysis of Case A. In Section 3, we shall state our results on the other cases omitting the proofs, as the proofs are similar.

We need the following definitions.

Definition 1. If the equation $f_{n}(z)-c=0$ has a multiple root, then $c$ is called a critical point of the inverse function $f_{-n}(z)$.

Definition 2. The immediate attractive set $A^{*}(\alpha)$ of a first order attractive fixpoint $\alpha$ is the maximal domain of normality of $\left\{f_{n}(z)\right\}$ which contains $\alpha$. The attractive set $A(\alpha)$ of $\alpha$ is defined by

$$
A(\alpha)=\left\{z \mid \lim _{n \rightarrow \infty} f_{n}(z)=\alpha\right\} .
$$

A modern survey of the main results of iteration theory can be found in Brolin [4]. We shall refer the reader to this paper for the results from iteration theory needed for our proofs. 


\section{On the iteration of $f(z)=z^{4}+p z$}

Let $f(z)=z^{4}+p z$, where $p$ is real. Then $f(z)$ has four finite first order fixpoints, $q_{1}, q_{2}, q_{3}$ and $q_{4}$, and $f_{-1}(z)$ has three finite critical points, $c_{1}, c_{2}$, and $c_{3}$. These are

$$
\begin{gathered}
q_{1}=0, \quad q_{2}=(1-p)^{1 / 3}, \quad q_{3}=q_{2} w, \quad q_{4}=q_{2} w^{2}, \\
c_{1}=-\frac{3 p}{4}\left(\frac{p}{4}\right)^{1 / 3}, \quad c_{2}=c_{1} w, \quad c_{3}=c_{1} w^{2},
\end{gathered}
$$

where $w$ and $w^{2}$ are the complex cube roots of unity.

Lemma 1.1. If $|p| \leqq 1$, then $c_{1}, c_{2}, c_{3} \in A^{*}(0)$.

Proof. $q_{1}=0$ is an attractive fixpoint for $|p|<1$ and a rationally indifferent one for $|p|=1$; at least one critical point belongs to $A^{*}(0)$ [4, Theorem 3.1, p. 109]. Suppose $c_{1} \in A^{*}(0)$. Since $c_{2}=c_{1} w$ and $c_{3}=c_{1} w^{2}$ and $z \in A^{*}(0)$ implies that $w z \in A^{*}(0)$, the result follows.

Lemma 2. The polynomial equation $g(t)=t^{7}\left(t^{3}-4\right)^{3}+27(4 t-3)=0$ has only one negative real root.

Proof. Since $g(0)<0$ and $g(-\infty)=\infty$, there is at least one negative real root. Clearly if there are more than one negative roots, there must be at least three. This would imply that $g^{\prime \prime}(t)=0$ will have at least one negative real root. But $g^{\prime \prime}(t)=$ $48 t^{5}\left(t^{3}-1\right)\left(t^{3}-4\right)\left(5 t^{3}-14\right)>0$ for $t<0$. Hence the lemma follows.

Lemma 3. If $-4 \theta / 3 \leqq p \leqq 4 / 3 \cdot 4^{1 / 3}$, then $c_{1}, c_{2}, c_{3} \notin A(\infty)$. Otherwise

We first prove

$$
c_{1}, c_{2}, c_{3} \in A(\infty)
$$

Lemma 4. If $-4 \theta / 3 \leqq p \leqq 0$, then

$$
\left|f\left(c_{1}\right)\right| \leqq q_{2}
$$

and

$$
\left|f_{2}(x)\right| \leqq q_{2} \text { if }|f(x)| \leqq q_{2} .
$$

Proof. First we consider (1). Since $p \leqq 0$, we have $f\left(c_{1}\right) \geqq 0$, and so (1) holds if $f\left(c_{1}\right) \leqq q_{2}$, i.e. if

$$
\left(c_{1}^{4}+p c_{1}\right)^{3} \leqq 1-p,
$$

i.e. if $g(t) \leqq 0$, where $t=3 p / 4$ and

$$
g(t)=t^{7}\left(t^{3}-4\right)^{3}+27(4 t-3) .
$$

By Lemma 2, $g(t) \leqq 0$ if $-4 \theta / 3<p \leqq 0$. Thus (1) is proved.

Next we prove (2). We have

$$
f_{2}(x)-q_{2}=\left\{f(x)-q_{2}\right\} h[f(x)] \text {, where } h(y)=y^{3}+q_{2} y^{2}+q_{2}^{2} y+1 \text {. }
$$


We claim that $h[f(x)]>0$ for every $x$. For, since the discriminant of $h^{\prime}(y)$ is negative, $h(y)=0$ has only one real root and $h(\gamma)$ is an increasing function of $y$. Denote the real root of $h(y)=0$ by $\alpha$. Hence the real zeros of $h[f(x)]$ are the real roots of the equation $f(x)=\alpha$, i.e. $x^{4}+p x-\alpha=0$. The minimum value of $x^{4}+p x-\alpha$ is $c_{1}-\alpha$. We assert that $c_{1}-\alpha>0$. If not, let $c_{1} \leqq \alpha$. Then $h\left(c_{1}\right)<h(\alpha)$ since $h(y)$ is increasing, i.e. $h\left(c_{1}\right) \leqq 0$. But

$$
h\left(c_{1}\right)=\frac{f\left(c_{1}\right)-q_{2}}{c_{1}-q_{2}}>0
$$

since both $f\left(c_{1}\right)-q_{2}$ and $c_{1}-q_{2}$ are negative when $-4 \theta / 3<p \leqq 0$, which is a contradiction. Hence $c_{1}-\alpha>0$, showing that no real root of $x^{4}+p x-\alpha=0$ exists, i.e. $h[f(x)]$ has no real zeros, and since $h[f(0)]=1$, it follows that $h[f(x)]>0$ for all $x$.

Since $f_{2}(x)-q_{2}=\left(f(x)-q_{2}\right) h[f(x)]$, we have $f_{2}(x) \leqq q_{2}$ if $|f(x)| \leqq q_{2}$. We show next that $f_{2}(x) \geqq-q_{2}$. Now,

$$
f_{2}(x)+q_{2}=[f(x)]^{4}+p[f(x)]+q_{2} \geqq 0
$$

if $27 p^{4}-256(1-p)<0$, which is obvious when $-4 \theta / 3<p \leqq 0$, i.e. $f_{2}(x) \geqq-q_{2}$ and Lemma 4 is proved.

Remark. The approximate value of $4 \theta / 3$ is 1.401 .

\section{Proof of Lemma 3}

We prove the lemma in four steps.

Step I. $p<-4 \theta / 3$

For $x>q_{2}$ we have $f(x)>x$ and hence $x \in A(\infty)$. If $p<4 \theta / 3$, then $g(t)>0$, i.e. $f\left(c_{1}\right)>q_{2}$. It follows that $c_{1} \in A(\infty)$. Since $\left|f_{n}\left(c_{1}\right)\right|=\left|f_{n}\left(c_{2}\right)\right|=\left|f_{n}\left(c_{3}\right)\right|$, it follows that $c_{1}, c_{2}, c_{3} \in A(\infty)$.

Step II. $-4 \theta / 3 \leqq p \leqq 0$

When $p=-4 \theta / 3, g(t)=0$, i.e. $f\left(c_{1}\right)=q_{2}$ and hence $f_{n}\left(c_{1}\right)=q_{2}$ for every $n$. If $-4 \theta / 3<p \leqq 0$, by Lemma $4\left|f\left(c_{1}\right)\right| \leqq q_{2}$ and $|f(x)| \leqq q_{2}$ implies $\left|f_{2}(x)\right| \leqq q_{2}$, i.e. $\left|f_{n}\left(c_{1}\right)\right| \leqq q_{2}$ for every $n$. Thus $c_{1}, c_{2}, c_{3} \notin A(\infty)$.

Step III. $0<p<(4 / 3) \cdot 4^{1 / 3}$

We claim that $\left|f_{n}\left(c_{1}\right)\right| \leqq\left|c_{1}\right|$ for every $n$. We have $f\left(c_{1}\right)=\left(c_{1} / 3\right)\left(4 t-t^{4}\right)$, where $p=4 t / 3$. Since $0<\left(4 t-t^{4}\right) / 3 \leqq 1$ when $0<t \leqq 4^{1 / 3}$, it follows that $\left|f\left(c_{1}\right)\right| \leqq\left|c_{1}\right|$. Also $f\left(c_{1}\right)=c_{1} \mu_{1}$, where $0<\mu_{1} \leqq 1$. Now, $f_{2}\left(c_{1}\right)=\left(c_{1} / 3\right)\left(4 \mu_{1} t-\mu_{1}^{4} t^{4}\right)$ and hence $\left|f_{2}\left(c_{1}\right)\right| \leqq\left|c_{1}\right|$. Similarly, $\left|f_{n}\left(c_{1}\right)\right| \leqq\left|c_{1}\right|$ for every $n$, i.e. $c_{1}, c_{2}, c_{3} \notin A(\infty)$. 
Step $I V \cdot p>(4 / 3) \cdot 4^{1 / 3}$

If $p>(4 / 3) \cdot 4^{1 / 3}$, then $f(x)>x$ for $x>0$ and hence $x \in A(\infty)$. Now $f\left(c_{1}\right)>0$ if $p>(4 / 3) \cdot 4^{1 / 3}$, and it follows that $c_{1}, c_{2}, c_{3} \in A(\infty)$. The lemma is completely proved.

Theorem 1. Let $f(z)=z^{4}+p z$ with p real.

i) If $-4 \theta / 3 \leqq p \leqq(4 / 3) \cdot 4^{1 / 3}$, then $F$ is connected. Otherwise $F$ is totally dis. connected and $m_{2} F=0$, where $m_{2}$ denotes the planar measure of $F$.

ii) $F$ is a Jordan curve if and only if $|p|<1$.

iii) $F \subset\left\{z|| z \mid \leqq(1+|p|)^{1 / 3}\right\}$.

Proof. i) If $-4 \theta / 3 \leqq p \leqq(4 / 3) \cdot 4^{1 / 3}$, then by Lemma $3 c_{1}, c_{2}, c_{3} \notin A(\infty)$, and if $p \notin\left[-4 \theta / 3,(4 / 3) \cdot 4^{1 / 3}\right]$, then $c_{1}, c_{2}, c_{3} \in A(\infty)$. The result then follows from Theorems 11.2 and 11.4 of Brolin [4, p. 125].

ii) By Lemma 1, $C_{1} \subset A^{*}(0)$ for $|p|<1$, and it follows from Theorem 11.3 that $F$ is a Jordan curve.

Now let $|p| \geqq 1$. If $p \geqq 1$, the rays $z=r, z=r e^{2 i \pi / 3}$ and $z=r e^{-2 i \pi / 3}, 0<r<\infty$, all belong to $A^{*}(\infty)$ for in all cases $f(z)=r^{4}+p r>|z|$ and hence $\left|f_{n}(z)\right| \rightarrow \infty$. Similarly, when $p \leqq-1$, the rays $z=-r, z=r e^{i \pi / 3}$ and $z=r e^{-i \pi / 3}, 0<r<\infty$, belong to $A^{*}(\infty)$. In this case $|f(z)|=r^{4}+|p| r>|z|$ and $\left|f_{n}(z)\right| \rightarrow \infty$.

It is clear that when $|p| \geqq 1,0 \in F$, and so by the symmetry of iteration $F$ cannot be a Jordan curve.

iii) If $|z|>(1+|p|)^{1 / 3}$, then $|f(z)|>|z|$ and hence $z \in A(\infty)$, i.e.

$$
F \subset\left\{z|| z \mid \leqq(1+|p|)^{1 / 3}\right\}
$$

This completes the proof of the theorem.

\section{Other cases}

In this section we state our results on the remaining cases mentioned in Section 1 . We omit the proofs.

Theorem 2. Let $f(z)=z^{4}+r$, r real.

i) If $-2^{1 / 3} \leqq r \leqq 3 /\left(4 \cdot 4^{1 / 3}\right)$, then $F(f)$ is connected. Otherwise $F(f)$ is totally disconnected and $m_{2} F(f)=0$, where $m_{2}$ denotes the planar measure of $F(f)$.

ii) $F(f)$ is a Jordan curve if and only if $-5 /\left(4 \cdot 4^{1 / 3}\right)<r \leqq 3 /\left(4 \cdot 4^{1 / 3}\right)$.

Theorem 3. If $f(z)=z^{4}+r, r$ real, then the iterates $f_{n}(z)$ have only constant limit functions in their domain of normality.

Theorem 4. Let $f(z)=z^{4}+p z^{2}$, p real. Then:

i) $F(f)$ is connected if and only if $-2(\sqrt{2}+1)^{1 / 3} \leqq p \leqq 2(\sqrt{2}-1)^{1 / 3}$. 
ii) $F(f)$ is a Jordan curve if and only if $-3 / 4^{1 / 3}<p<2(\sqrt{2}-1)^{1 / 3}$.

iii) If $-5 / 12^{1 / 3}<p<-3 / 4^{1 / 3}$ and $p \neq-2$, then $F(f)$ contains an infinite number of Jordan curves.

Theorem 5. If $f(z)=z^{4}+p z^{2}$, p real, then the iterates $f_{n}(z)$ have only constant limit functions in their domains of normality.

Theorem 6. Let $f(z)=z^{4}-p z^{2}+\sqrt{p}, p \geqq 0$. Then:

i) $F(f)$ is connected if and only if $0 \leqq p \leqq 4$. If $p \geqq 4, F(f)$ is real and $F(f) \subset$ $[-\sqrt{p}, \sqrt{p}]$. If $p=4, F(f)$ is the interval $[-2,2]$.

ii) $F(f)$ is a Jordan curve if and only if $0 \leqq p<1 / 4^{1 / 3}$.

Theorem 7. Let $f(z)=z^{4}-p z^{2}+\sqrt{p}, p \geqq p$. If $p>4$, then $F(f)$ contains infinite number of single point components.

Theorem 8. If $f(z)=z^{4}-p z^{2}+\sqrt{p}, p>0$, then the iterates $f_{n}(z)$ have only constant limit functions in their domains of normality.

Theorem 9. Let $f(z)=z^{4}+p z^{2}+r z+s, p, q, r, s$ reals. If $r^{2} / 8+p^{3} / 27 \leqq 0$ and $(r-1)^{2}<4 p s$, then $F(f)$ is totally disconnected and $m_{2} F=0$, where $m_{2}$ denotes the planar measure. Further, $f_{n}(z)$ have only constant limit functions in their domains of normality.

The authors wish to thank Dr. I. N. Baker of Imperial College, London, for his comments in the preparation of this paper.

\section{References}

[1] BAKER, I. N.: Limit functions and sets of non-normality in iteration theory. - Ann. Acad. Sci. Fenn. Ser. A I Math. 467, 1970, 1-11.

[2] Bhattacharyya, P.: On the set of normality and iteration of $e^{2}-1$. - Publ. Math. Debrecen 19, 1972, 29-34.

[3] Bhattacharyya, P., and Y. E. Arumaraj: On the structure of the Fatou set for the polynomial $z^{3}+p z, p<-3$. - Math. Rep. Toyama Univ. 1980, 1:3, 123-141.

[4] Brolin, H.: Invariant sets under iteration of rational functions - Ark. Mat. 6, 1965-67, 104-144.

[5] Fatou, M. P.: Sur les équations fonctionnelles. - Bull. Soc. Math. France 47, 1919, 161-271 and 48, 1920, 33-94.

[6] Fatou, M. P.: Sur l'itération des fonctions transcendant esentières. - Acta Math. 47, 1926, 337 370.

[7] Van Haselen, A.: Sur l'itération de $\log (1+z)$. - Enseign. Math. 30, 1931, 267-271.

[8] Julia, G.: Mémoire sur l'itération des fonctions rationnelles. - J. Math. Pures Appl. (8) 1, $1918,47-245$.

[9] Myrberg, P. J. Iteration der reellen Polynome zweiten Grades. - Ann. Acad. Sci. Fenn. Ser. A I Math. 256, 1958, 1-10.

[10] Myrberg, P. J.: Iteration der reellen Polynome zweiten GradesII. - Ibid. 268, 1959, 1-13. 
[11] Myrberg, P. J.: Sur l'itération des polynomes réels quadratiques. - J. Math. Pures Appl. (9) 41, 1962, 339-351.

[12] Myrberg, P. J.: Iteration der reellen Polynome zweiten Grades III. - Ann. Acad. Sci. Fenn. Ser. A I Math. 336/3, 1963, 1-18.

[13] Myrberg, P. J.: Iteration der Binome beliebigen Grades. - Ibid. 348, 1964, 1-14.

[14] TopfFER, H.: Über die Iteration der ganzen transzendenten Funktionen insbesondere von sin und cos. - Math. Ann. 117, 1940, 65-84.

Indian Institute of Technology

Department of Mathematics

Madras 600036

India

Received 8 September 1980

Revision received 23 February 1981 